\title{
Liver Specific Alkaline Phosphatase Measurement
}

National Cancer Institute

\section{Source}

National Cancer Institute. Liver Specific Alkaline Phosphatase Measurement. NCI

Thesaurus. Code C119267.

The determination of the amount of the liver specific alkaline phosphatase in a biological specimen. 